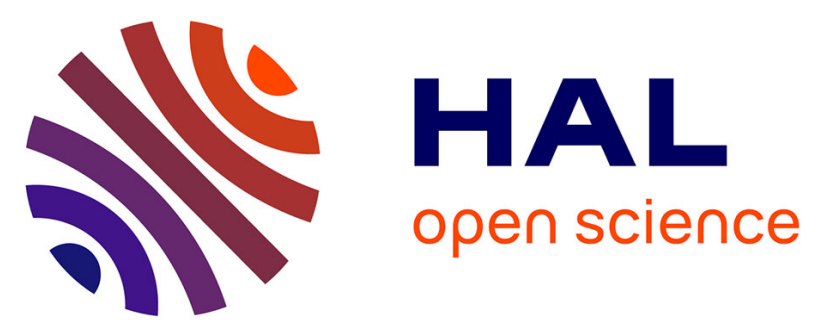

\title{
Intermittent collective dynamics of domain walls in the creep regime
}

Pablo Matías Grassi, Alejandro Kolton, Vincent Jeudy, Alexandra Mougin, Sebastian Bustingorry, Javier Curiale

\section{- To cite this version:}

Pablo Matías Grassi, Alejandro Kolton, Vincent Jeudy, Alexandra Mougin, Sebastian Bustingorry, et al.. Intermittent collective dynamics of domain walls in the creep regime. Physical Review B: Condensed Matter and Materials Physics (1998-2015), 2018, 98, 10.1103/PhysRevB.98.224201 . hal02331839

\section{HAL Id: hal-02331839 \\ https://hal.science/hal-02331839}

Submitted on 24 Oct 2019

HAL is a multi-disciplinary open access archive for the deposit and dissemination of scientific research documents, whether they are published or not. The documents may come from teaching and research institutions in France or abroad, or from public or private research centers.
L'archive ouverte pluridisciplinaire HAL, est destinée au dépôt et à la diffusion de documents scientifiques de niveau recherche, publiés ou non, émanant des établissements d'enseignement et de recherche français ou étrangers, des laboratoires publics ou privés. 


\title{
Intermittent collective dynamics of domain walls in the creep regime
}

\author{
Matías Pablo Grassi, ${ }^{1}$ Alejandro B. Kolton, ${ }^{1,2}$ Vincent Jeudy, ${ }^{3}$ Alexandra Mougin, ${ }^{3}$ \\ Sebastian Bustingorry, ${ }^{4}$ and Javier Curiale ${ }^{1,4}$ \\ ${ }^{1}$ Instituto Balseiro, Universidad Nacional de Cuyo-CNEA, Av. Bustillo 9500, 8400 S. C. de Bariloche, Río Negro, Argentina \\ ${ }^{2}$ CONICET, Centro Atómico Bariloche, 8400 San Carlos de Bariloche, Río Negro, Argentina \\ ${ }^{3}$ Laboratoire de Physique des Solides, CNRS, Univ. Paris-Sud, Université Paris-Saclay, 91405 Orsay, France \\ ${ }^{4}$ Instituto de Nanociencia y Nanotecnología, CNEA-CONICET, Centro Atómico Bariloche, \\ 8400 San Carlos de Bariloche, Río Negro, Argentina
}

(Received 6 June 2018; published 6 December 2018)

\begin{abstract}
We study the ultraslow domain-wall motion in ferromagnetic thin films driven by a weak magnetic field. Using time-resolved magneto-optical Kerr effect microscopy, we access to the statistics of the intermittent thermally activated domain-wall jumps between deep metastable states. Our observations are consistent with the existence of creep avalanches: roughly independent clusters with broad size and ignition waiting-time distributions, each one composed by a large number of spatiotemporally correlated thermally activated elementary events. Moreover, we evidence that the large-scale geometry of domain walls is better described by depinning rather than equilibrium universal exponents.
\end{abstract}

DOI: 10.1103/PhysRevB.98.224201

\section{INTRODUCTION}

Domain walls (DWs) in thin ferromagnetic films have become a paradigmatic system [1-6] to learn about the universal interplay between disorder, elasticity, and thermal fluctuations in driven interfaces. Such physics is relevant for a large variety of experimental systems [7-9] and for potential applications, as DWs are building blocks for proposed magnetic storage devices [10]. The caveat is that even an arbitrarily weak disorder has a rather dramatic effect on the DW dynamics, notably the occurrence of a depinning threshold [11,12]. Below the threshold, DWs are pinned at zero temperature and they present a thermally activated glassy behavior called the creep regime at finite temperature. A better understanding of the impact of disorder in low-velocity regimes is thus fundamental for a comprehensive study of DW dynamics and of disordered elastic interfaces in general.

Most of the experimental studies on weakly driven DW motion, including very recent ones [2-6], focused on the universal features of the steady DW mean velocity vs the field $H$ and temperature $T$, but not in its spatiotemporal fluctuations. Such kind of study has been mostly performed close to the depinning threshold, where the fluctuations are dominated by large deterministic collective events. For example, avalanchesize distribution and its universal properties has been discussed in the context of Barkhausen noise [13], contact lines of liquids [7], crack propagation [14], and even in reaction fronts in disordered flows [8] and active cell migration [9]. Well below the depinning threshold, the phenomenology of avalanches have remained much less clear [15]. Recently, however, theoretical studies of ultraslow creep motion [16] have unveiled rather unexpected and nontrivial spatiotemporal patterns, whose elementary events (EEs) strongly differ from those encountered close to the depinning threshold. Therefore, tackling experimentally a detailed statistical study of magnetization reversal events is particularly interesting.
The numerical simulations reported in Ref. [16] show that creep motion of a one dimensional interface model proceeds via a sequence of EEs of fluctuating sizes. These EEs are the minimal thermally activated jumps that make the DW overcome energy barriers and irreversibly advance under the applied field $H$. The size statistics of EEs display broad distributions, with a characteristic lateral size cutoff $L_{\mathrm{opt}} \sim H^{-3 / 4}$ and a characteristic area size $S_{\mathrm{opt}} \sim L_{\mathrm{opt}}^{5 / 3}$. These results confirm the existence of an optimal "thermal nucleous" as proposed in the pioneer creep theories [11,12]. Since energy barriers for DW motion scale as $U_{\mathrm{opt}} \sim L_{\mathrm{opt}}^{1 / 3} \sim$ $H^{-1 / 4}$ (Ref. [17]), Arrhenius activation of these nuclei leads to the celebrated creep-law $\ln v \sim-H^{-1 / 4} / T$ for the mean velocity $v$ at which the DW move under the action of a small magnetic field $H$. The EEs are not normally distributed in size and are not independent as traditionally assumed. On one hand, below $S_{\text {opt }}$, EE areas are power-law distributed as $P_{\mathrm{EE}}(S) \approx S^{-\tau_{\mathrm{EE}}} G\left(S / S_{\mathrm{opt}}\right)$, with $\tau_{\mathrm{EE}}$ a characteristic exponent and $G(x)$ a rapidly decaying function for $x>1$. On the other hand, EEs tend to cluster in space and time, forming larger cluster events (CEs). These CEs are similar to the so-called "creep avalanches" suggested by functional renormalization group calculations in Ref. [17] and experimentally noticed in Ref. [15]. Such composite objects are, unlike EEs, weakly correlated and have a much broader distribution of areas, $P_{\mathrm{CE}}(S) \sim S^{-\tau_{\mathrm{CE}}}$ with $\tau_{\mathrm{CE}}$ a universal exponent. These interesting predictions were not yet evidenced experimentally nor confirmed by other theoretical approaches.

In this paper, we test the above scenario by a statistical analysis of the ultraslow time evolution of magnetization reversal in ferromagnetic $\mathrm{Pt} / \mathrm{Co} / \mathrm{Pt}$ thin films. For different time windows of duration $\Delta t$, we determine the size $(S)$ distribution $P_{\mathrm{WE}}(S) \equiv P_{\mathrm{WE}}(S ; \Delta t, T, H)$ of the observed consecutive compact magnetization reversal area that we call a window event (WE). This procedure permits us to relate WEs with EEs and CEs and to show that the features displayed by 
$P_{\mathrm{WE}}(S)$ are consistent with the picture summarized above of rare localized EEs acting as epicenters of large CEs or "creep avalanches," each made of a large number of spatiotemporally correlated EEs. Furthermore, our analysis of the intermittent collective DW motion allows us to characterize the statistics of waiting times between epicenter EEs, thus going beyond the "geometric" predictions of Ref. [16].

\section{METHODS}

Experiments were mainly performed on a $\operatorname{Pt}(4.5 \mathrm{~nm})$ / $\operatorname{Co}(0.7 \mathrm{~nm}) / \mathrm{Pt}(3.5 \mathrm{~nm})$ thin ferromagnetic film with perpendicular magnetic anisotropy. A polar magneto-optical Kerr effect (PMOKE) microscope was used to image magnetic domains. To characterize the DW dynamics, starting with a seed magnetic domain, a train of magnetic field pulses of duration $t$ and intensity $H$ were applied perpendicular to the film plane to favor the growth of the initial domain. The DW velocity was then computed following a standard differential protocol. After identifying the creep regime in the $H-T$ plane by fitting the creep-law $\ln v \sim-H^{-1 / 4} / T$, we fix $T$ to two possible values, room temperature and $50^{\circ} \mathrm{C}$, and choose $H=46.1 \mathrm{Oe}$ and $H=24.2 \mathrm{Oe}$, respectively, such that $v \sim$ $1 \mathrm{~nm} \mathrm{~s}^{-1}$ in each case. We then analyze the magnetization reversal events at each temperature, for a total applied field time $t=27000 \mathrm{~s}$. Since the characteristic areas of EEs are expected to scale as $S_{\text {opt }} \sim H^{-5 / 4}$, and the energy barriers for nucleation as $U_{\text {opt }} \sim H^{-1 / 4}$, choosing fields deep in the creep regime allows us to maximize, in principle, our spatial and temporal sensitivity to intrinsic collective events. For these fields, we indeed observe a clear intermittent (i.e., not smooth) growth. To characterize it statistically, during the long-time magnetic field pulse we stroboscopically observe the growth at intervals $\Delta t$, such that $t \gg \Delta t$. The duration $\Delta t$ is much larger than the acquisition time of each image, and much smaller than the pulse time $t$ so to collect a large number of events. This allows us to compute their area distribution, $P_{\mathrm{WE}}(S)$, for different $\Delta t$ and $T$. Although we mainly report results for one region of a specific sample, we have also performed less detailed but similar measurements in other regions of the same sample and also in a different material and checked robustness of our results. We discard WEs touching any border of the region of interest to not underestimate their area and make a proper comparison with theoretical predictions. We have checked that this protocol does not affect the tails of $P_{\mathrm{WE}}(S)$ for the time windows $\Delta t$ used. We refer the reader to Appendix A for further details on our experimental setup and protocols. Magnetization reversal events were previously obtained in irradiated $\mathrm{Pt} / \mathrm{Co} / \mathrm{Pt}$ samples [15], identifying between 30 and 50 events depending on field values. In the present work, we were able to obtain thousands of WEs, thus allowing a more precise statistical description, amenable to comparison with the universal theoretical predictions.

\section{RESULTS}

\section{A. Domain-wall motion within the creep regime}

The obtained field dependence of DW velocity for the analyzed sample is presented in Fig. 1(a). The figure shows the evolution of the velocity as a function of the magnetic
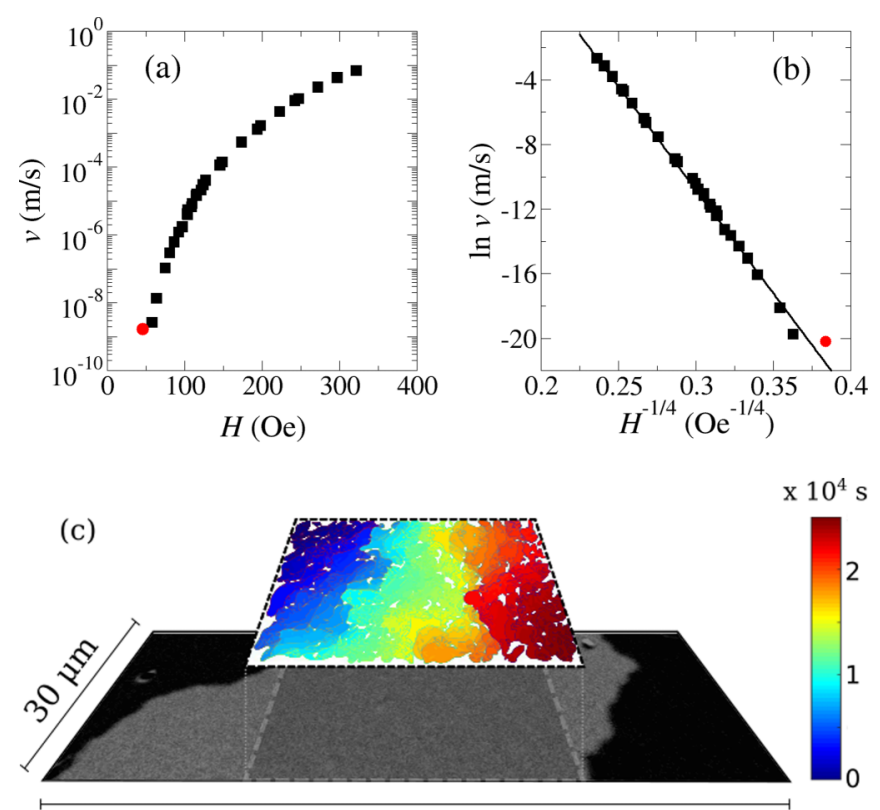

$90 \mu \mathrm{m}$

FIG. 1. (a), (b) Velocity-field characteristics for ultrathin $\mathrm{Pt} / \mathrm{Co} / \mathrm{Pt}$ magnetic thin film in different scales. In (b), the fitted solid line confirms agreement with the creep velocity law: $\ln v \sim H^{-1 / 4}$. The red point corresponds to a single long pulse of duration $t=$ $27000 \mathrm{~s}$ at a small field of $H=46.1 \mathrm{Oe}$ and room temperature (RT). The total reversed area over this long pulse is indicated in (c) and corresponds to $v=1.710^{-9} \mathrm{~m} / \mathrm{s}$. During this long pulse, PMOKE images were taken every $t_{0}=15 \mathrm{~s}$, allowing us to identify $N_{\text {WE }}\left(t_{0}, t\right)=1151$ magnetization reversal events or "window events" (WEs), highlighted over the image. The color scale corresponds to the time at which each WE was observed.

field over eight orders of magnitude. Within the creep regime, thermal activation over a field-dependent energy barrier leads to a stretched exponential increase of the velocity, given by $[1,11,12,17]$

$$
v=v_{0} \exp \left[-\frac{T_{d}}{T}\left(\frac{H}{H_{d}}\right)^{-\mu}\right],
$$

where $v_{0}$ is a temperature-dependent velocity [4], $T$ the temperature, $k_{B} T_{d}$ a typical energy scale coming from the competition between elasticiy and disorder $\left(k_{B}\right.$ being the Boltzmann constant), $H_{d}$ the depinning field and $\mu=1 / 4$ the universal creep exponent. As shown in Fig. 1(b), a straight line with a negative slope in a plot of $\ln v$ against $H^{-1 / 4}$ confirms that the measured velocities are within the creep regime, and in addition that the system belongs to the universality classes of one dimensional elastic systems displacing in a two dimensional media, with a random-bond type of disorder and short-range elasticity. The fit to the creep formula of Eq. (1) for the two temperatures we analyzed are

$$
\begin{aligned}
\ln \left[v \mathrm{~m}^{-1} \mathrm{~s}\right] & =-128(1)(\mathrm{Oe})^{1 / 4} H^{-1 / 4}+27.6(3), \\
& =-100(2)(\mathrm{Oe})^{1 / 4} H^{-1 / 4}+24.4(5),
\end{aligned}
$$


TABLE I. Characteristic depinning values for the studied temperature and field values: $H_{d}$ is the depinning field, $T_{d}$ is the depinning temperature, $H_{d}^{1 / 4} \frac{T_{d}}{T}$ is the slope of the creep plot [see Eqs. (2) and (3)], and $T / T_{d}$ and $\left(\frac{T}{T_{d}}\right)\left(\frac{H}{H_{d}}\right)^{1 / 4}$ are related to the distribution of waiting times as discussed in Sec. III E.

\begin{tabular}{lcc}
\hline \hline$T[\mathrm{~K}]$ & 293 & 323 \\
\hline$H[\mathrm{Oe}]$ & 46.1 & 24.2 \\
$T_{d}[\mathrm{~K}]$ & 7142 & 6369 \\
$H_{d}[\mathrm{Oe}]$ & 760 & 650 \\
$H_{d}^{1 / 4} \frac{T_{d}}{T}\left[\mathrm{Oe}^{1 / 4}\right]$ & 128 & 100 \\
$\frac{T}{T_{d}}$ & 0.04 & 0.06 \\
$\left(\frac{T}{T_{d}}\right)\left(\frac{H}{H_{d}}\right)^{1 / 4}$ & 0.02 & 0.02 \\
\hline \hline
\end{tabular}

at $T=\mathrm{RT}$ [Eq. (2)] and $T=50^{\circ} \mathrm{C}$ [Eq. (3)]. This data, the experimental estimates for the depinning field $H_{d}$, the depinning temperature $T_{d}$ and key characteristic scales are reported in Table I.

With the aim of pursuing the characterization of small magnetization reversal events responsible of the creep motion of elastic systems, one should consider that the typical area size $S_{\text {opt }}$ of the "optimal thermal nuclei" responsible for the velocity of Eq. (1) dramatically increases when the magnetic field decreases, as $S_{\mathrm{opt}} \propto H^{-v_{\mathrm{eq}}\left(1+\zeta_{\mathrm{eq}}\right)}$, with $v_{\mathrm{eq}}$ and $\zeta_{\text {eq }}$ positive universal exponents $[1,11,12,17]$. Since velocity follows an streched exponential dependence with $S_{\text {opt }}[11,12]$, decreasing the magnetic field implies to perform very long time experiments. Therefore, after nucleation of a single domain, a small magnetic field $(H=46.1 \mathrm{Oe})$ is applied during a single long time pulse $(t=27000 \mathrm{~s}$, i.e., 7.5 hours), reaching a velocity $v=1.710^{-9} \mathrm{~m} / \mathrm{s}$. The velocity-field data thus obtained is indicated as a red point in Fig. 1(b), and the differential image shown in Fig. 1(c) corresponds to the full displacement of the DW under these conditions. To identify magnetically reversed regions (WEs), during the total long pulse time $t$, PMOKE images were taken every $t_{0}=15 \mathrm{~s}$, which corresponds to the minimum time window $\Delta t$. During the image acquisition the magnetic field remained always ON. Consecutive images were subtracted and, since the velocity is small $\left(v=1.710^{-9} \mathrm{~m} / \mathrm{s}\right)$, most of differential images do not show a clear advance of the DW. Eventually, a magnetization reversal region resulting in a local advance of the DW position is observed. After the whole long-time pulse experiment, the total reversed area [indicated in Fig. 1(c)] is fragmented into many small spatially compact regions obtained from the subtraction of consecutive images taken after $t_{0}$. The number of WEs is $N_{\mathrm{WE}}\left(t_{0}, t\right)=1151$ and are highlighted with a color code over the image of the reversed area in Fig. 1(c). Due to the characteristics of the used PMOKE microscope and the image analysis, the smallest detectable displacement of the DWs correspond to events close to $0.3 \mu \mathrm{m}^{2}$ (25 pixels).

\section{B. Event areas}

In Fig. 2, we show typical WE sequences, for four different values of $\Delta t$, from a $15 \mathrm{~s}$ to $120 \mathrm{~s}$. We can appreciate that, for a given growth, each $\Delta t$ induces a particular partition of the total reversed area of the sequence. At large $\Delta t$, the
$\Delta t=15 \mathrm{~s}$

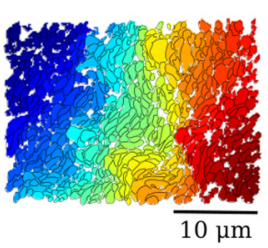

$60 \mathrm{~s}$

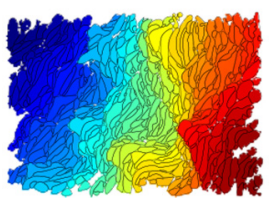

$30 \mathrm{~s}$

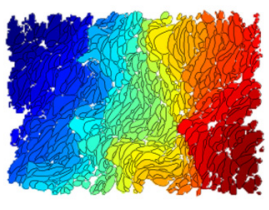

$\mathrm{x} 10^{4} \mathrm{~s}$

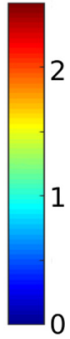

FIG. 2. Sequences of magnetization reversal areas (WEs) detected for different time windows of duration $\Delta t$, for $T=\mathrm{RT}$ and $H=46.1$ Oe. The color scale corresponds to the time at which each WE, delimited by contours lines, was detected.

coalescence of several smaller WEs corresponding to smaller $\Delta t$ becomes evident.

In Figs. 3(a) and 3(b), we compare size distributions $P_{\mathrm{WE}}(S)$, from $\Delta t=15$ to $180 \mathrm{~s}$ at room temperature $T=$ RT and a field $H=46.1 \mathrm{Oe}$, and from $\Delta t=20$ to $160 \mathrm{~s}$ at $T=50^{\circ} \mathrm{C}$ and a field $H=24.2 \mathrm{Oe}$, respectively. The first remarkable feature of all these distributions is their
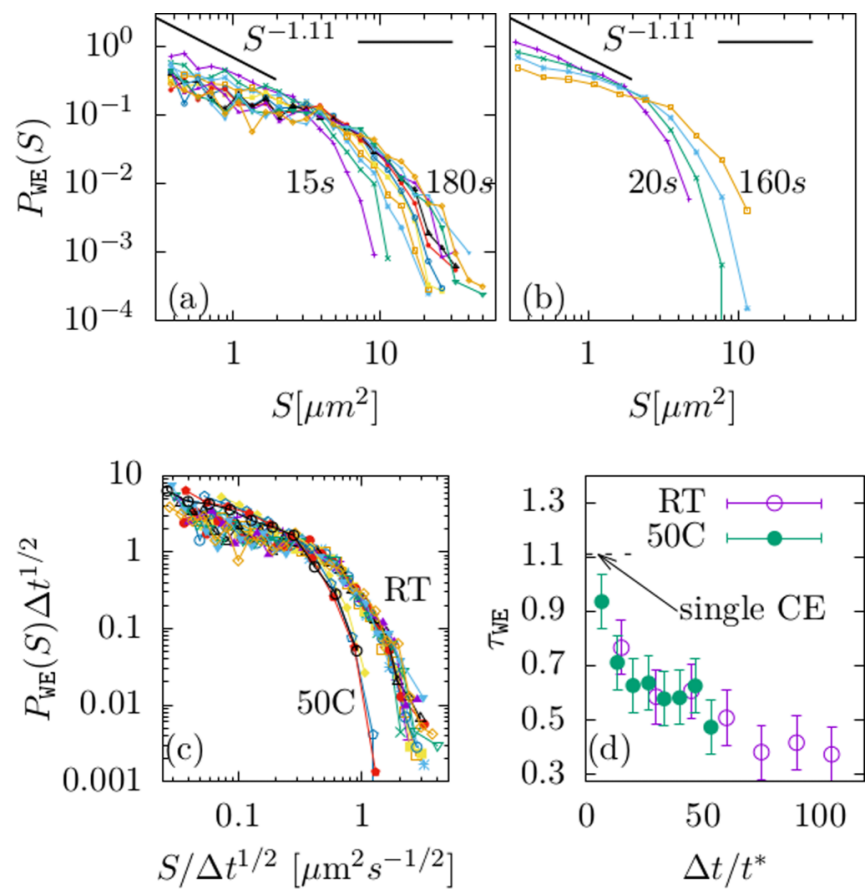

FIG. 3. WE area distributions for increasing window times $\Delta t$ (as indicated) at RT and $H=46.1 \mathrm{Oe}$ (a) and at $T=50^{\circ} \mathrm{C}$ and $H=$ $24.2 \mathrm{Oe}$ (b). In both cases, $v \sim 1 \mathrm{~nm} \mathrm{~s}^{-1}$. At small $S$, we compare the initial decay of $P_{\mathrm{WE}}(S)$ with $S^{-\tau_{\mathrm{CE}}}$, with $\tau_{\mathrm{CE}} \approx 1.11$, where $\tau_{\mathrm{CE}}$ corresponds to depinning avalanches. (c) The collapse scaling shows that the data of (a) and (b) displays a large size cutoff scaling $S_{\mathrm{WE}} \sim\left(\Delta t / t^{*}\right)^{1 / 2}$, with $t^{*}$ an $H$ - and $T$-dependent characteristic time. (d) Effective power-law exponents $\tau_{\mathrm{WE}}$ for $P_{\mathrm{WE}}(S)$ vs $\Delta t / t^{*}$. 
broadness, which can be roughly described by $P_{\mathrm{WE}}(S)=$ $S^{-\tau_{\mathrm{WE}}} G_{\mathrm{WE}}\left(S / S_{\mathrm{WE}}\right)$, where $\tau_{\mathrm{WE}}$ is an effective power-law exponent and $S_{\mathrm{WE}}$ the cutoff value such that the function $G_{\mathrm{WE}}(x)$ is constant for small $x$ and decays faster than a power-law for $x \gtrsim 1$. Quantitatively similar size-distributions were observed in different regions of the same sample and also in other kinds of magnetic films (see Appendix A).

Both $\tau_{\mathrm{WE}}$ and the large-size cutoff $S_{\mathrm{WE}}$ depend on $\Delta t$. As can be appreciated in Figs. 3(a)-3(b), $S_{\mathrm{WE}}$ increases with $\Delta t$, more specifically $S_{\mathrm{WE}} \sim\left(\Delta t / t^{*}\right)^{1 / 2}$. The fair collapse of $P(S) \Delta t^{1 / 2}$ vs $S / \Delta t^{1 / 2}$ shown in Fig. 3(c) confirms this dependence. Here, $t^{*} \equiv t^{*}(T, H)$ is a characteristic time. Concomitantly, in Fig. 3(d) we show that $\tau_{\mathrm{WE}} \approx 1$ for the smallest $\Delta t / t^{*}$ for the whole data of Figs. 3(a) and 3(b). Note also that the same $t^{*}$ that describes the $S_{\mathrm{WE}}(T, H)$ dependence allows us to build a master curve for $\tau_{\mathrm{WE}}$ vs $\Delta t / t^{*}$. For the characteristic times $t^{*}$, we find $t_{50 C}^{*} \approx 1 \mathrm{~s}$ at $T=50^{\circ} \mathrm{C}, H=24.2 \mathrm{Oe}$, and $t_{R T}^{*} \approx t_{50 C}^{*} / 3$ at $T=\mathrm{RT}$, $H=46.1$ Oe. Therefore, $S_{\mathrm{WE}} \approx(\Delta t)^{1 / 2} \mu \mathrm{m}^{2} \mathrm{~s}^{-1 / 2}$ in the first case, and $S_{\mathrm{WE}} \approx(3 \Delta t)^{1 / 2} \mu \mathrm{m}^{2} \mathrm{~s}^{-1 / 2}$ in the second one.

Since EEs of Ref. [16] are power-law distributed with an exponent $\tau_{\mathrm{EE}} \approx 1.17$, it is tempting to directly compare small $\Delta t$ WEs, which are also typically small, to EEs. A rough estimate for the $\mathrm{Pt} / \mathrm{Co} / \mathrm{Pt}$ films we study shows that the largest EEs are of the order of $S_{\text {opt }}=10^{-3}\left(H_{d} / H\right)^{1.25} \mu \mathrm{m}^{2}$, where $H_{d}$ is the depinning field. ${ }^{1}$ Since $H_{d} \approx 637 \mathrm{Oe}$, and our lowest field is $H=46 \mathrm{Oe}$, we get that $S_{\text {opt }} \sim 10^{-5} \mu \mathrm{m}^{2}$, which is clearly well below our PMOKE resolution of roughly $0.3 \mu \mathrm{m}^{2}$ (25 pixels). We thus conclude that our detected WE cannot be a single EE, but the sum of a large number of them. Namely, if in a time window $\Delta t$ we have $\mathcal{N}_{\mathrm{EE}}$ such events, of sizes $s_{1}, s_{2}, \ldots, s_{\mathcal{N}_{\mathrm{EE}}}$, compactly grouped in a WE, its random area is $\mathcal{S}_{\mathrm{WE}} \approx \sum_{i=1}^{\mathcal{N}_{\mathrm{EE}}} s_{i}$. The statistics of $\mathcal{S}_{\mathrm{WE}}$ thus directly relates to the statistics of EE random sizes $s_{i}$ contributing to the same $\mathrm{WE}$ and of their $\Delta t$-dependent and fluctuating number $\mathcal{N}_{\mathrm{EE}}$.

Given the small area of the EE compared to our detected WE, a pure statistical analysis is convenient. If the EE were considered independent and accumulating at a well-defined rate on each WE, by virtue of the central limit theorem, we would naively expect $P_{\mathrm{WE}}(S)$ to develop an approximate Gaussian shape around $\overline{\mathcal{N}_{\mathrm{EE}}} \bar{S}$. $P_{\mathrm{WE}}(S)$ shows no tendency to approximate a normal nor even a peaked distribution; however, it is broad, even for $\Delta t$ in the minutes timescale. To interpret this it is worth recalling that the central limit theorem tell us that $\mathcal{S}_{\mathrm{WE}} \approx \sum_{i=1}^{\mathcal{N}_{\mathrm{EE}}} s_{i}$ should converge to a Gaussian distribution if $\mathcal{N}_{\mathrm{EE}}$ is large enough and the $s_{i}$ have finite variance and short-ranged correlations [18]. The EEs have finite variance and, although they appear to be spatially correlated, there is no evidence of correlation between their areas [16]. We hence interpret that $\mathcal{N}_{\mathrm{EE}}$ must be a strongly fluctuating quantity for all the $\Delta t$ analyzed. Indeed, we experimentally observe for a fixed $\Delta t$ well-defined bursts of magnetic activity, with $\mathcal{S}_{\text {WE }} \gg 0.3 \mu \mathrm{m}^{2}$, coexisting with WEs in the resolution edge $\mathcal{S}_{\text {WE }} \gtrsim 0.3 \mu \mathrm{m}^{2}$, at the same $H$ and $T$. Since any PMOKEresolved area $\mathcal{S}_{\mathrm{WE}}>0.3 \mu \mathrm{m}^{2}$ has a large number of EEs, we

\footnotetext{
${ }^{1}$ See Supplemental Material in Ref. [16].
}
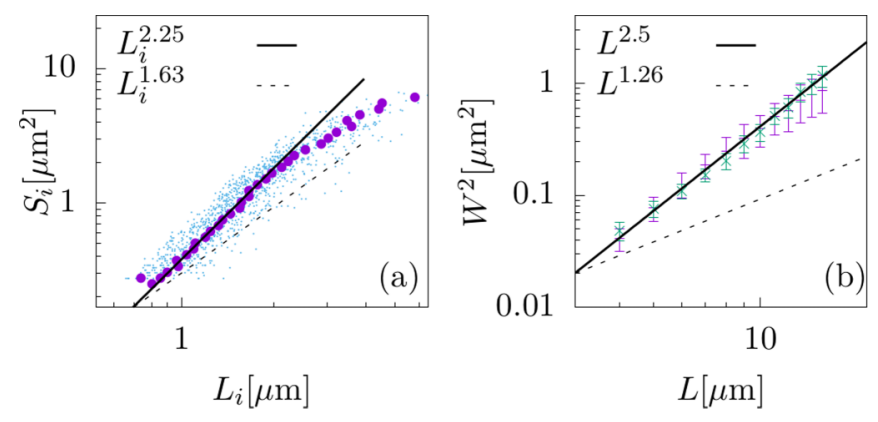

FIG. 4. (a) Aspect ratio scaling of $\Delta t=15 \mathrm{~s}$ WEs. The solid (dashed) line shows the expected depinning scaling $S_{i} \sim L_{i}^{1+\zeta_{d}}$ for qEW (qKPZ) class. (b) Scaling of the square width $W^{2}$ of DW segments of size $L$, for two typical configurations at RT. The solid (dashed) line shows the expected qEW (qKPZ) scaling at depinning, $W^{2} \sim L^{2 \zeta_{d}}$, with $\zeta_{d}=1.25(0.63)$.

arrive to the first important observation of our paper: EEs are strongly clustered spatiotemporally.

\section{Domain-wall roughness}

The results of the previous section are consistent with the EEs clustering predicted for simple DW models [16,19]. To go beyond, since EEs are too small to be experimentally resolved, one is immediately tempted to compare our experimentally resolved WEs with the predicted CEs. Indeed, unlike EEs, CEs are not expected [16] to be strongly correlated, as we also observe for WEs. Moreover, the predicted value for $\tau_{\mathrm{CE}} \approx 1.11$ is only slightly above $\tau_{\mathrm{WE}} \approx 1$ observed in Fig. $3(\mathrm{~d})$ for the smallest $\Delta t$. To argue that WE may indeed approach the single intrinsic $\mathrm{CE}$ in the small $\Delta t$ limit, we start by noting that the same scaling of zero-temperature depinning avalanches, $S_{i} \sim L_{i}^{1+\zeta_{d}}$, is also expected for CEs [16] at finite temperature. In Fig. 4(a), we analyze for $T=$ RT the approximately oblong shapes of WEs by plotting the areas $S_{i}$ of each WE sampled from a long sequence, versus their corresponding lateral size $L_{i}$, defined as the major axis length of the reversed blobs. A crossover is observed at $S \approx 2 \mu \mathrm{m}^{2}$, below which we observe a $S_{i} \sim L_{i}^{2.25}$ scaling. ${ }^{2}$ The two main candidate depinning universality classes that are consistent with the observed creep law $\ln v \sim H^{-1 / 4} / T$ are the 1D quenchedEdwards-Wilkinson (qEW), and the 1D quenched KardarParisi-Zhang (qKPZ). The first predicts $\zeta_{d} \approx 1.25$ [20] while the second $\zeta_{d} \approx 0.63$ [21-23]. Only the qEW value is in good quantitative agreenment with Fig. 4(a), in the small size WE limit. ${ }^{3}$ In addition, Fig. 3(d) is quantitatively consistent with the relation $\tau_{\mathrm{CE}}=2-2 /\left(1+\zeta_{d}\right) \approx 1.11$ predicted for qEW. To investigate this issue, in Fig. 4(b) we computed the squared width $W^{2}(L) \equiv \overline{u_{L}^{2}(x)}-\overline{u_{L}(x)}{ }^{2}$ from different

\footnotetext{
${ }^{2}$ We show both the original noisy $S_{i}$ vs $L_{i}$ curve and a smoothed curve, obtained by grouping similar areas in small bins and by assigning the average of $L_{i}$ to each area bin. Indistinguishable results are obtained by binning $L_{i}$ rather than $S_{i}$.

${ }^{3}$ The downward deviation crossover for large WEs or lateral acceleration is due to coalescence effects in the proximity of a percolation transition. See Sec. III G for further details.
} 
small segments of size $L$ extracted from typical DW configurations, where $u_{L}(x)$ is the DW displacement measured with respect to the untilted segment (see Appendix B details). The scaling $W^{2} \sim L^{2 \zeta_{d}}$ is consistent with the qEW depinning roughness exponent $\zeta_{d}=1.25$ and thus with Figs. 3(d) and 4(a). We then arrive to the second important observation of our paper: WEs approach single CEs in the small $\Delta t$ limit and we find experimental evidence that the DW roughness and CE statistical properties are better described by depinning rather than equilibrium exponents as theoretically predicted in Refs. [17,24,25]. A smaller roughness exponent $0.69 \pm 0.07$ was observed in extended DWs in the same material in the pioneering work by Lemerle et al. [1], and interpreted to be the equilibrium exponent $\zeta_{\text {eq }}=2 / 3$. Such interpretation implies an observation scale below $L_{\text {opt }}$ [24]. However, from Ref. [1], we infer $L_{\mathrm{opt}} \approx 0.18 \mu \mathrm{m}$, lower than their PMOKE resolution of $0.28 \mu \mathrm{m}$. We thus conclude that a spatial crossover from the $\mathrm{qEW}$ value $\zeta_{d} \approx 1.25$ to a nonequilibrium exponent $\sim 0.69 \pm 0.07$ must exist. A natural candidate is the quenched Kardar-Parisi-Zhang (qKPZ) or directed percolation depinning exponent $\zeta_{d} \approx 0.63$.

\section{Event lengths}

In Fig. 5(a), we show the area $S_{i}$ vs the major axis length $L_{i}$ of each WE. The difference with Fig. 4(a), where $\Delta t=15 \mathrm{~s}$, is that now we plot WEs for all $\Delta t$, from $15 \mathrm{~s}$ to $180 \mathrm{~s}$ to observe the effects of large WEs. We show both the cloud obtained from raw data and an averaged version by grouping areas in small logarithmically increasing bins and by taking the corresponding average value of $L_{i}$ in such groups. We compare with the depinning scaling $S_{i} \sim L_{i}^{1+\zeta_{d}}$, expected for CEs in the creep regime [16], both for the qEW class where $\zeta_{d}=1.25$ and for the qKPZ class, where $\zeta_{d} \approx 0.63$. At $L_{i} \approx$ $L^{*}=2 \mu \mathrm{m}$, a clear crossover is observed (indicated by the vertical line). As can be appreciated in the figure, for $L_{i}<L^{*}$ a better agreement is obtained for $\mathrm{qEW}$, as compared, for instance, with the qKPZ class.

In Fig. 5(b), we show the (nonnormalized) probability distribution $P_{\mathrm{WE}}(L)$ for all the $L_{i}$ observed. As for $P_{\mathrm{WE}}(S)$, we observe a broad distribution. If WEs were a part, single, or dominated by a single CE, we expect, indeed, WEs to display power-law distributions similar to the ones observed for depinning avalanches, $P_{\mathrm{CE}}(S) \sim S^{-\tau_{\mathrm{CE}}}$ and $P_{\mathrm{CE}}(L) \sim S^{-\tau_{L}}$, where $\tau_{\mathrm{CE}}$ and $\tau_{L}$ are related to depinning exponents. The general exponents are well known [26]:

$$
\begin{gathered}
\tau_{\mathrm{CE}}=2-\left(\zeta_{d}+1 / \nu_{d}\right) /\left(1+\zeta_{d}\right), \\
\tau_{L}=\tau_{\mathrm{CE}}\left(1+\zeta_{d}\right)-\zeta_{d},
\end{gathered}
$$

where $\zeta_{d}$ is the depinning roughness exponent and $v_{d}$ the depinning correlation length exponent. For the $\mathrm{qEW}$ universality class, we have $\zeta_{d} \approx 1.25$ and, by virtue of the statistical tilt symmetry [27], $v_{d}=1 /\left(2-\zeta_{d}\right) \approx 1.33$. On the other hand, $\zeta_{d} \approx 0.63$ and $\nu_{d} \approx 1.73$ for the qKPZ class [23], where the statistical tilt symmetry is broken. This yields $\tau_{\mathrm{CE}} \approx 1.11$, $\tau_{L} \approx 1.25$ for the $\mathrm{qEW}$ class, and $\tau_{\mathrm{CE}} \approx 1.25, \tau_{L} \approx 1.42$ for the qKPZ class. The effective power law at intermediate $L \lesssim L^{*}$ (indicated by the vertical line) is roughly consistent
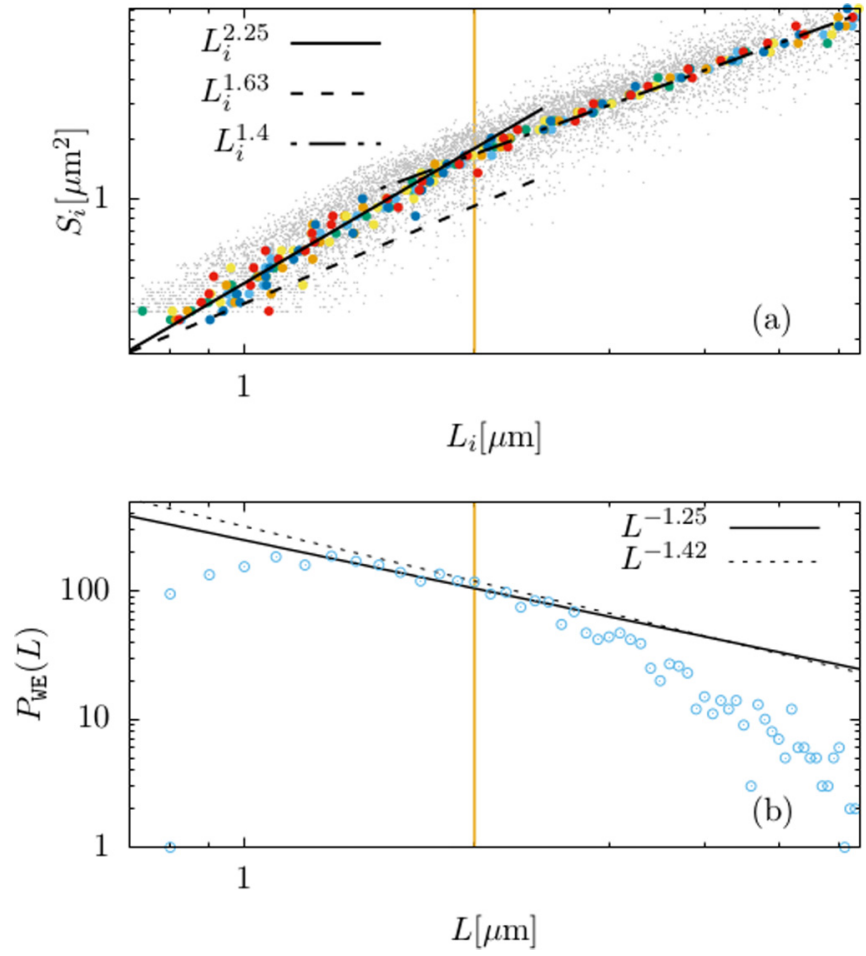

FIG. 5. (a) Aspect-ratio of WEs obtained experimentally, between the area $S_{i}$ and major axis length $L_{i}$ of individual WE. We display the data $S_{i}$ vs $L_{i}$ for WEs corresponding to all values of $\Delta t$ to enhance the crossover behaviour and access large WEs. Small WEs fairly scale as CEs or depinning avalanches in the qEW class, $S_{i} \sim$ $L_{i}^{1+\zeta_{d}}$ with $\zeta_{d} \approx 1.25$ and thus $S_{i} \sim L_{i}^{2.25}$ (solid line), as compared to the qKPZ class, with $\zeta_{d}=0.63$, predicting $S_{i} \sim L_{i}^{1.63}$ (dashed line). The dotted-dashed line indicates a fair $S_{i} \sim L_{i}^{1.4}$ scaling, which can be rationalized using a simple model. (b) Probability distribution (nonnormalized) for the major axis length $L_{i}$ of the events shown in (a). In (a) and (b) a vertical line indicates the approximate location of the aspect-ratio crossover, $L^{*}$.

with qEW. Unfortunately, however, the effective power law observed in Fig. 5(b) is roughly consistent with both classes, unlike Fig. 5(a), which is more consistent with the qEW class.

The crossover at $L^{*}$, observed in Fig. 5(a), may be associated to the CE coalescence process occurring for large WEs. ${ }^{4}$ In that case, a WE area can be written as a sum of a given number $\mathcal{N}_{\mathrm{CE}}$ of CE areas, $\mathcal{S}_{\mathrm{WE}} \approx \sum_{i=1}^{\mathcal{N}_{\mathrm{CE}}} \mathcal{S}_{i}$. Since $P_{\mathrm{CE}}(S)$ is a broad distribution, the typical WE area is dominated by the largest areas and thus relates to the typical number of $\mathrm{CE}$ as $S_{\mathrm{WE}} \sim N_{\mathrm{CE}}^{1 /(\tau-1)}$. The lateral size of a WE instead satisfies an inequality $L_{\mathrm{WE}}<\sum_{i=1}^{\mathcal{N}_{\mathrm{CE}}} \mathcal{L}_{i}$, as the fluctuating CE lateral extensions $\mathcal{L}_{i}$ can now overlap. Since $P_{\mathrm{CE}}(L)$ is also a broad distribution, we can use the same extreme value argument to estimate $L_{\mathrm{WE}} \lesssim N_{\mathrm{CE}}^{1 /\left(\tau_{L}-1\right)}$. Combining these results, we

\footnotetext{
${ }^{4}$ In our protocol, the maximum WE lateral size $L_{i}$ was limited by the lateral size $L_{\text {roi }}$ of the region of interest. We did not consider WEs touching nor spanning completely the region of interest. If spanning WEs were considered, we would have $S_{\mathrm{WE}} \approx v \Delta t L_{\text {roi }}$ in the long-time limit, with $v$ the average velocity, shown in Figs. 1(a) and $1(\mathrm{~b})$.
} 
get $S_{\mathrm{WE}} \gtrsim L_{\mathrm{WE}}^{\left(\tau_{L}-1\right) /(\tau-1)} \equiv L_{\mathrm{WE}}^{1+\zeta_{d}}$. This shows that WE areas should scale with their length approximately as CE in the $L_{i}<L^{*}$ regime, as observed in Fig. 5(a). Above $L^{*}$, however, where large WEs become a nonnegligible fraction of the interface, the last scaling prediction breaks down. In Sec. III G, we discuss a simple model that quantitatively accounts for the crossover observed at $L^{*}$ in the $S_{i}$ vs $L_{i}$ plot.

\section{E. Waiting times}

The behavior at large $\Delta t$, where the probability to observe single CE in a WE decreases, is directly related to the behavior of the large-size $P_{\mathrm{WE}}(S)$ cutoff, $S_{\mathrm{WE}}$, with $\Delta t$. In such regime we can regard each WE area as the sum of a given number $\mathcal{N}_{\mathrm{CE}}$ of cluster areas, $\mathcal{S}_{\mathrm{WE}}=\sum_{i=1}^{\mathcal{N}_{\mathrm{CE}}} \mathcal{S}_{i}$. As $\mathcal{N}_{\mathrm{CE}}$ can only grow irreversibly with $\Delta t$, so does the large-size cutoff $S_{\mathrm{WE}}$. Naively, one may think that $S_{\mathrm{WE}}$ should linearly increase with $\Delta t$ because the sum of all WE areas observed in a region of a fixed lateral size $L$ should grow as $L v \Delta t$ in a steady-state regime. As shown in Fig. 3(c), we find instead a sublinear increase $S_{\mathrm{WE}} \sim\left(\Delta t / t^{*}\right)^{1 / 2}$. To make sense of this striking observation, it is instructive to regard the area $\mathcal{S}_{\mathrm{WE}}$ vs $\Delta t$ as a continuous-time continuous-jump random walk, with random $\mathrm{CE}$ area increments $\mathcal{S}_{i}$ and waiting times $\delta_{i}$ for the ignition of a new CE, such that $\Delta t=\sum_{i=1}^{\mathcal{N}_{\mathrm{CE}}} \delta_{i}$. If we assume that the $\delta_{i}$ are distributed according to $\psi(\delta) \sim t^{* \alpha} \delta^{-(1+\alpha)}$, with $0<\alpha \leqslant 1$ characterizing the broadness of $\psi(\delta)$, we get $\Delta t \sim t^{*} N_{\mathrm{CE}}^{1 / \alpha}$ for the typical number of events $N_{\mathrm{CE}}$ in a $\Delta t$. Since the same heuristic arguments apply for the broadly distributed CEs, we get $S_{\mathrm{WE}} \sim N_{\mathrm{CE}}^{1 /\left(\tau_{\mathrm{CE}}-1\right)}$. Combining the two last results, we get $S_{\mathrm{WE}} \sim\left(\Delta t / t^{*}\right)^{\alpha /\left(\tau_{\mathrm{CE}}-1\right)}$, which fairly describes our data of Fig. 3(c) if $\alpha /\left(\tau_{\mathrm{CE}}-1\right) \approx 1 / 2$. Using $\tau_{\mathrm{CE}} \approx 1$.11, we obtain $\alpha \approx 0.05$.

Broad waiting-time distributions have been heuristically derived for creep motion [28,29], borrowing ideas from more general random energy models (see, for instance, Ref. [18]), and also observed numerically close to the depinning threshold. ${ }^{5}$ The basic idea is to assume that the EE barrier distribution behaves as $P(U) \sim \exp \left[-U / U^{*}\right] / U^{*}$ for a large barrier $U$, with $U^{*}$ a characteristic energy (with $U$ and $U^{*}$ in units of temperature). If temperature is small enough, the typical time to overcome $U$ is given by the Arrhenius law, $\delta \sim t^{*} \exp [U / T]$, with $t^{*}$ a characteristic time. Changing variables, we obtain $\psi(\delta) \sim \alpha t^{* \alpha} \delta^{-(1+\alpha)}$, with $\alpha \sim T / U^{*}$. Since clustering implies that not all EEs have the same $U$, we will argue that the $\delta_{i}$ corresponds to the special EEs that act as CE epicenters. These EEs may be associated to the ones allowing us to escape from dominant configurations [25]. Two different predictions for $U^{*}$ and thus for $\alpha$ are found in the literature. In Ref. [28], it is assumed that $U^{*} \equiv T_{d}$. With $T_{d}$ from Table I, we obtain $\alpha=0.04$ for $T=293 \mathrm{~K}$ and $\alpha=0.06$ for $T=$ $323 \mathrm{~K}$. Both results are in excellent agreement with our data, which gives $\alpha \approx 0.05$. In Ref. [29], on the other hand, the characteristic energy is taken as the optimal nucleus barrier $U^{*} \equiv T_{d}\left(H_{d} / H\right)^{\mu}$, with $\mu=1 / 4$ for the one dimensional elastic string. The exponent is thus again nonuniversal but

\footnotetext{
${ }^{5}$ E. A. Jagla, unpublished.
}

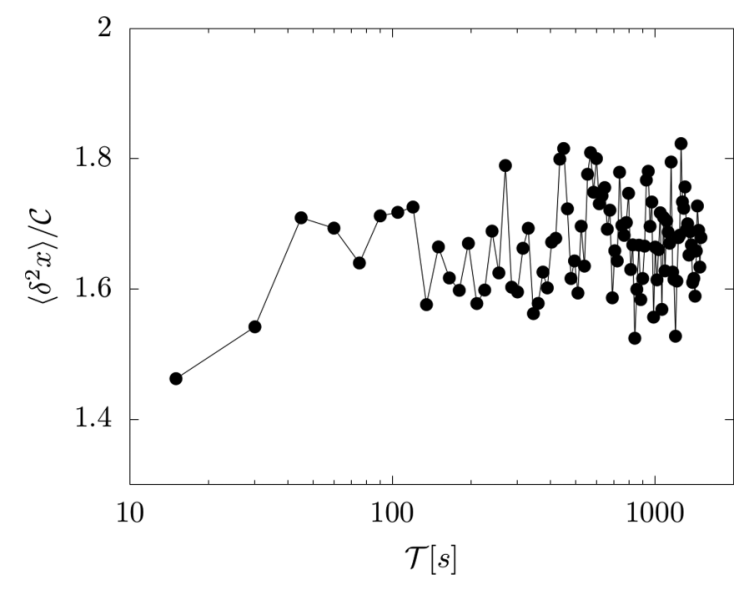

FIG. 6. Normalized mean square distance $\left\langle\delta^{2} x(\mathcal{T})\right\rangle / \mathcal{C}$ as a function of $\mathcal{T}$, measured at $T=\mathrm{RT}$ and $H=46.1 \mathrm{Oe}$.

now it is also field dependent, $\alpha \approx\left(T / T_{d}\right)\left(H / H_{d}\right)^{\mu}$. From Table I, we obtain $\alpha \approx 0.02$ both for the two temperatures and their corresponding fields. This value is only slightly below but is again of the order of $\alpha \approx 0.05$ we infer from our measurements. Both predictions are in rough agreement with the empirical $\alpha \approx 0.05$ we obtain from the time-scaling of $S_{\mathrm{WE}}$ we observe in Fig. 3(c) for the two temperatures. It would be interesting to perform a more systematic study as a function of $T$ and $H$ to further test these theories. The previous observations lead us to argue that WEs give access not only to the CE area (at small $\Delta t$ ) but also to the waitingtime statistics (at larger $\Delta t$ ). As CEs start at a seed EE, the $\delta_{i}$ must be controlled by their energy barrier distribution. ${ }^{6}$

\section{F. Event correlations}

To further test the connection between WEs and CEs we have also studied correlations from the spatiotemporal correlations of the registered positions $x_{i}$ of the $N$ measured WE epicentres. To do that we used the mean square distance $\left\langle\delta^{2} x(\mathcal{T})\right\rangle \equiv \sum_{i=1}^{N}\left[x_{i+n}-x_{i}\right]^{2} / N$, which depends only on the temporal separation $\mathcal{T}=n t_{0}$. For noncorrelated WE epicentre sequences, $\left\langle\delta^{2} x(\mathcal{T})\right\rangle$ tends to a constant value $\mathcal{C}=\left(L_{0}+\right.$ 1) $\left(L_{0}+2\right) / 2$, where $L_{0}$ is approximately the lenght of the DW in units of the spatial discretization. ${ }^{7}$ Figure 6 shows $\left\langle\delta^{2} x(\mathcal{T})\right\rangle / \mathcal{C}$ measured at $T=\mathrm{RT}$ and $H=46.1$ Oe. One can see that even for short $\mathcal{T}$ it becomes approximately constant as expected for uncorrelated events (note that $\left\langle\delta^{2} x(\mathcal{T})\right\rangle / \mathcal{C}>1$ for large $\mathcal{T}$ due to an underestimation of the length of DW). We hence conclude that WEs are very weakly correlated in sharp contrast with the predicted EE correlations in Ref. [16] and consistent to what is predicted for CEs and more generaly for depinning avalanches. This observation further confirms our identification of WEs with single CEs or with coalesced groups of them, for small or large $\Delta t$, respectively.

\footnotetext{
${ }^{6}$ Barrier distributions for dominant metastable states have been computed in Ref. [25] for forces below and near $f_{c}$. For small systems a roughly exponential right tail followed by a cut-off can be appreciated.

${ }^{7}$ L. Foini, A. Rosso, private communication.
} 


\section{G. Heuristic model for large WEs}

Summing up, our results are consistent with the predictions of Ref. [16] after identifying the small $\Delta t$ WEs with the predicted CEs. At large $\Delta t$ WEs cannot be single CEs, however, and deviations from the predicted properties for CEs are expected. This is already apparent in Fig. 5(a), where large WEs display a clearly different length to area aspect ratio than the expected for CEs. Moreover, Fig. 5(a) shows a clear crossover from the expected $S_{i} \sim L_{i}^{1+\zeta_{d}} \mathrm{CE}$ behavior to a different behavior, rather well described by a new powerlaw, $S_{i} \sim L_{i}^{1.4}$. There is no theoretical predictions yet for this crossover so we propose here a simple, heuristic model.

A very simple model can explain the behavior of $S_{i}$ vs $L_{i}$ observed experimentally, shown in Fig. 5(a). The idea is to think WE as the compact objects formed by random deposition of simulated CEs, with a lateral sizes $L_{i}$ sampled from $P_{\mathrm{CE}}(L) \sim L^{-\tau_{L}}$. We can also assume, for simplicity, that the corresponding areas satisfy a deterministic relation $S_{i}=$ $L_{i}^{1+\zeta_{d}}$ assumption that leads automatically to $P_{\mathrm{CE}}(S) \sim S^{-\tau_{\mathrm{CE}}}$, with $\tau_{L}, \tau_{\mathrm{CE}}$ and $\zeta_{d}$ related by Eqs. (4) and (5). Both assumptions are reasonable approximations according to creep simulations [16]. To simulate this model, we generated such events in the interval $[0,1]$, sequentially increasing the number of deposited CEs. The process starts with one WE, which equals the first deposited CE. Adding more CEs may produce more WEs (especially for a small number of deposited CEs) or can decrease their number due to the possible coalescence with an existing WE, if the new CE overlaps it. When a coalescence between a new $\mathrm{CE}$ and an existing WE takes place, the area of the resulting WE is the sum of the new $\mathrm{CE}$ area with the previous area of the WE, but the length of the new WE can either remain constant or increase at its left, right, or both corners simultaneously. In more rare cases, the new CE can overlap more than one WE. The process finishes when a single WE spans the whole interval, i.e., when the deposited CEs percolate the system. To make statistics over many sequences, at this point we reset the simulation and restart, adding a first CE into a new actogram. Figure 7(d) shows actograms corresponding to four runs.

To be concrete, for the simulations we use the values $\zeta_{d}=$ $1.25, \tau=1.11$ and $\tau_{L}=\zeta_{d}$, corresponding to the $1 \mathrm{D} \mathrm{qEW}$ depinning class. We sample the epicenter of each $\mathrm{CE}$ from a uniform distribution in the interval $[0,1]$ and its lateral size $l_{i}$ by $\left.l_{i}=0.05\left[r\left(l_{\max }^{\tau_{L}+1}-l_{\min }^{\tau_{L}+1}\right)+l_{\min }^{\tau_{L}+1}\right)\right]^{1 /\left(\tau_{L}+1\right)}$ with $l_{\min }=$ $10^{-5}$ and $l_{\max }=1.5$, and $r$ a different uniform random number in the interval $[0,1]$. This produces a power-law distribution $P_{\mathrm{CE}}(l) \sim l^{-\tau_{L}}$ with a cutoff at $l_{\max }$. The area of such a CE is simply $s_{i}=l_{i}^{1+\zeta_{d}}$. Using different parameters yields qualitatively similar results.

We now discuss the results of the model. In Fig. 7(a), we show that the model reproduces the main features observed experimentally in Fig. 5(a). Small WEs below a characteristic crossver scale display the $S_{i} \sim L_{i}^{2.25}$. This is natural, as most of the small WEs are individual CEs. At approximately $L^{*} \sim$ 0.07 , there is a crossover toward $S_{i} \sim L_{i}^{1.4}$ for large WEs. Remarkably, this new exponent is indistinguishable from the one we obtain experimentally [see Fig. 5(a)]. We leave for a future study to understand the origin and possible universality of this new exponent. It is worth noting, however, that the crossover may be associated to the lateral acceleration that
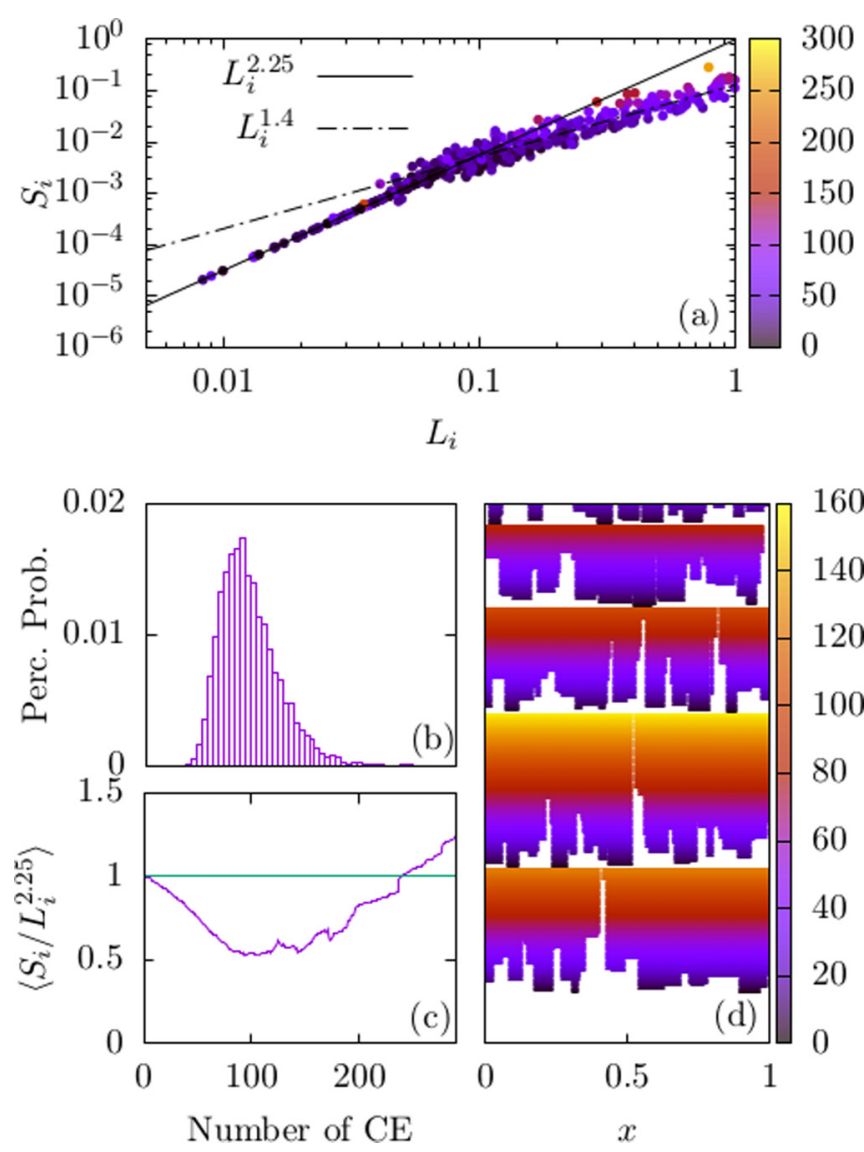

FIG. 7. A simple model for understanding the area vs major axis length of large WEs. (a) Simulation results for the model [compare them with experimental results in Fig. 5(a)]. The solid line indicates the qEW depinning scaling, and the dashed dotted line the effective power law observed at large WEs. The color bar indicates number of randomly deposited CEs. Results are reported in arbitrary units. (b) Probability distribution for percolation, as a function of the number of randomly deposited CEs. (c) Average aspect-ratio scaling versus number of CEs. (d) From bottom to top, four actograms showing the growth of compact WEs as we increase the number of deposited CEs (as indicated by the color bar). The actograms display segments centered at uniformly distributed epicenters, whose size equals the lateral extension of the growing WE. When the system percolates, i.e., a single WE spans the system (set to unity), the actogram is reset and a new deposition process starts.

occurs when the WEs almost percolate the $[0,1]$ interval. In Fig. 7(b), we show the probability to percolate as a function of the number of deposited CEs. Most of the points in Fig. 7(a) in the $S_{i} \sim L_{i}^{1.4}$ regime belong to states with a high probability to percolate. It is also worth noting that finite size effects, due to the finiteness of the interval and the broad range of the CE lateral size distribution, play an important role here. In Fig. 7(c), we show that the average anisotropic aspect-ratio $S_{i} / L_{i}^{2.25}$ is unity only for a small number of CEs, but then decreases, implying an accelerated lateral growth of WEs compared with the area growth. This effect may produce the crossover and the downward deviation appreciated in Fig. 7(a), and also experimentally shown in Fig. 5(a). For an even larger number of CEs, the aspect-ratio increases as large WEs tend to completely overlap with most of the new CEs 
and thus increase their areas without modifying their lateral size. Those states are near to percolate but need a rare CE to overlap the voids between the few remaining WEs.

The model presented has some unphysical features. In particular, the random deposition process implies, in the longtime limit, a growing interface with a nonstationary width. The model describes satisfactorily the crossover in $S_{i}$ vs $L_{i}$ observed in the experiments, however, so the necessary surface relaxation effects or correlations that may make the width to saturate are not relevant for the regime we aim to describe. In addition, the number of deposited CEs does not strictly represent time. Broadly distributed times between random depositions could be easily added, however, to further test the picture suggested by the experiments. Particularly, to reproduce the area and lateral size distributions as a function of the window time $\Delta t$ found experimentally.

\section{CONCLUSIONS}

From our results, the following picture emerges. Creep dynamics is driven by EEs with a broad size distribution and a large-size cutoff controlling the mean velocity. The seed EEs that trigger a cascade of extra EEs are separated by broadly distributed waiting times. Repeated, this collective process of ignition and correlated growth produce independent CEs statistically very similar to depinning avalanches, that may coalesce into larger compact objects. Hence, CEs can be truly regarded as "creep avalanches." The described picture, that drastically changes the naive view of creep motion as independent thermally nucleated displacements, is likely to appear not only in other magnetic films but in the creep regime of other disordered elastic systems in general.

\section{ACKNOWLEDGMENTS}

We thank E. Ferrero, A. Rosso, E. Jagla, G. Durin, T. Giamarchi, P. Le Doussal, L. Foini, V. Lecomte, and J. L. Barrat for enlightening discussions. We thank D. Jordan for his help on digitizing DWs. This work was partly supported by Grants No. PIP11220120100250CO/CONICET, No. PICT2016-0069/FONCyT, and No. UNCuyo 06/C490 and C017, from Argentina. The France-Argentina Project ECOS-Sud No. A12E03 is also acknowledged.

\section{APPENDIX A: SAMPLES \& EXPERIMENTAL PROTOCOL}

Experiments were mainly performed on a $\mathrm{Pt} / \mathrm{Co} / \mathrm{Pt}$ ultrathin ferromagnetic film, a prototypical system which has been the focus of many studies of DW motion [1,2,4,5,30-33]. The studied sample was a $\operatorname{Pt}(4.5 \mathrm{~nm}) / \mathrm{Co}(0.7 \mathrm{~nm}) / \operatorname{Pt}(3.5 \mathrm{~nm})$ thin film, with the thickness of each layer indicated in parenthesis. The film was sputter grown at $300 \mathrm{~K}$ on etched $\mathrm{Si} / \mathrm{SiO}_{2}$ substrate. The magnetic response of this system to an external out-of-plane magnetic field is characterized by a square magnetic cycle with a well defined remnant magnetization, typical of systems with perpendicular magnetic anisotropy [2].

PMOKE microscopy has been used to image magnetic domains after applying magnetic field pulses perpendicular to the film plane. After fully magnetizing the sample in one direction, a short pulse in the opposite direction and of
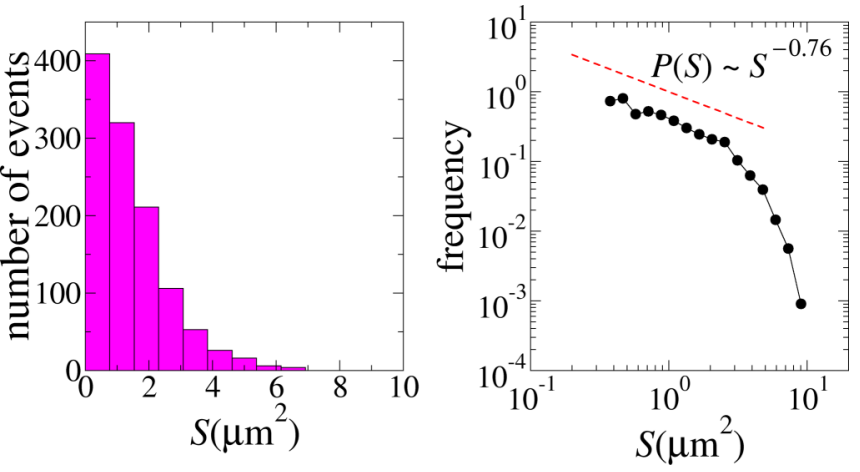

FIG. 8. Histograms for the $N_{\text {tot }}\left(t_{0}, t\right)=1151$ WEs shown in Fig. 1(c), obtained by comparing consecutive images taken every $\Delta t=t_{0}=15 \mathrm{~s}$ at $T=\mathrm{RT}$ and $H=46.1 \mathrm{Oe}$. The histogram with uniform binning is presented in the left panel, while in the right panel a logarithmic binning is used for the same WEs. Power-law behavior at small sizes can be described with $P_{\mathrm{WE}}(S) \sim S^{-\tau_{\mathrm{WE}}}$ with the distribution exponent $\tau_{\mathrm{WE}} \approx 0.76 \pm 0.1$.

intensity $H=130$ Oe was first applied to nucleate a seed magnetic domain. Then, a second pulse of duration $t$ and intensity $H$ was applied to favor the growth of the initial magnetic domain. DW velocity was then computed as the ratio between the linear advance of the DW linear advance $\Delta x$ and the pulse duration, $v=\Delta x / t$. Experiments were performed at $T=\mathrm{RT}$ (room temperature) and $T=50^{\circ} \mathrm{C}$. To measure velocities between $10^{-9} \mathrm{~m} / \mathrm{s}$ and $10^{-1} \mathrm{~m} / \mathrm{s}$, pulses of different amplitude and duration were used. In all cases, the total number of pulses was 15 or more and the rise time of the pulses was more than one order of magnitude faster than the pulse duration. The shortest pulse was $1 \mathrm{~ms}$ and the largest one $1800 \mathrm{~s}$. Due to the spatial resolution of our microscope, for velocities smaller than $10^{-8} \mathrm{~m} / \mathrm{s}$, we observed that there was no difference in the observed DW velocity if the magnetic field was ON or OFF during the image acquisition. In all the cases, given the illumination condition, the used shutter time of the camera was $200 \mathrm{~ms}$.

Although we mainly report results for one region of a specific sample, we have made similar measurements in other regions of the same sample and also in a $\mathrm{Pt}(6 \mathrm{~nm}) /[\mathrm{Co}(0.2 \mathrm{~nm}) / \mathrm{Ni}(0.6 \mathrm{~nm})]_{3} / \mathrm{Al}(5 \mathrm{~nm})$ sample, where the numbers in parenthesis stand for thickness and the ferromagnetic layer consists in a stack of three $\mathrm{Co}(0.2 \mathrm{~nm}) / \mathrm{Ni}(0.6 \mathrm{~nm})$ bilayers (for more information about these samples and their DW dynamics, see Refs. [34,35]). In both cases, the results are consistent with the main universal results reported for the specific region of the $\mathrm{Pt} / \mathrm{Co} / \mathrm{Pt}$ sample in Sec. III.

Magnetization reversal events were previously obtained in irradiated $\mathrm{Pt} / \mathrm{Co} / \mathrm{Pt}$ samples [15], identifying between 30 and 50 events depending on field values. In the present work, as we previously anticipated, we were able to obtain a large amount of WEs. This represents a quantitative progress in view of the fact that this allows us to perform a deeper statistical description of the data. Figure 8 shows the obtained histogram of the $1151 \mathrm{WE}$ areas shown in Fig. 1(c) by comparing consecutive images taken every $t_{0}=15 \mathrm{~s}$. Since we are seeking power-law-like distributions and their effective 
exponents, it is convenient to use logarithmic binning. In the right panel of Fig. 8, we use the same WEs used in the left panel to build a new histogram. In this case, dividing the number of events per interval by the width of the interval, the probability distribution is obtained. Figure 8 shows a powerlaw signature at small size values with a cutoff around $3 \mu \mathrm{m}^{2}$. The distribution is of the form $P_{\mathrm{WE}}(S)=S^{-\tau_{\mathrm{WE}}} G_{\mathrm{WE}}\left(S / S_{\mathrm{WE}}\right)$, where $\tau_{\mathrm{WE}}$ is the power-law exponent and $S_{\mathrm{WE}}$ the cutoff value such that the function $G_{\mathrm{WE}}(x)$ rapidly decays for $x \gtrsim 1$.

For a proper comparison with theoretical predictions, we discarded in the statistical analysis events touching the borders of the region of interest, i.e., the observation region, otherwise their area would be underestimated. This may affect, however, the tails of the size distribution, corresponding to large events, with a lateral size of the order or larger than the lateral size $L$ of the observation region. For the range of time windows $\Delta t$, we consider WEs of lateral size $L$ are extremely rare, however. Indeed, we observe a $\Delta t$-dependent but clearly $L$-independent cutoff in our distributions, growing as $\Delta t^{1 / 2}$ (with a temperature-dependent prefactor). Such $\Delta t$ dependence is used to estimate the waiting-time distribution exponent for cluster "ignition events". On the other hand, the power-law decay effective exponent of WEs, which is also central to our analysis and for the comparison with theory,
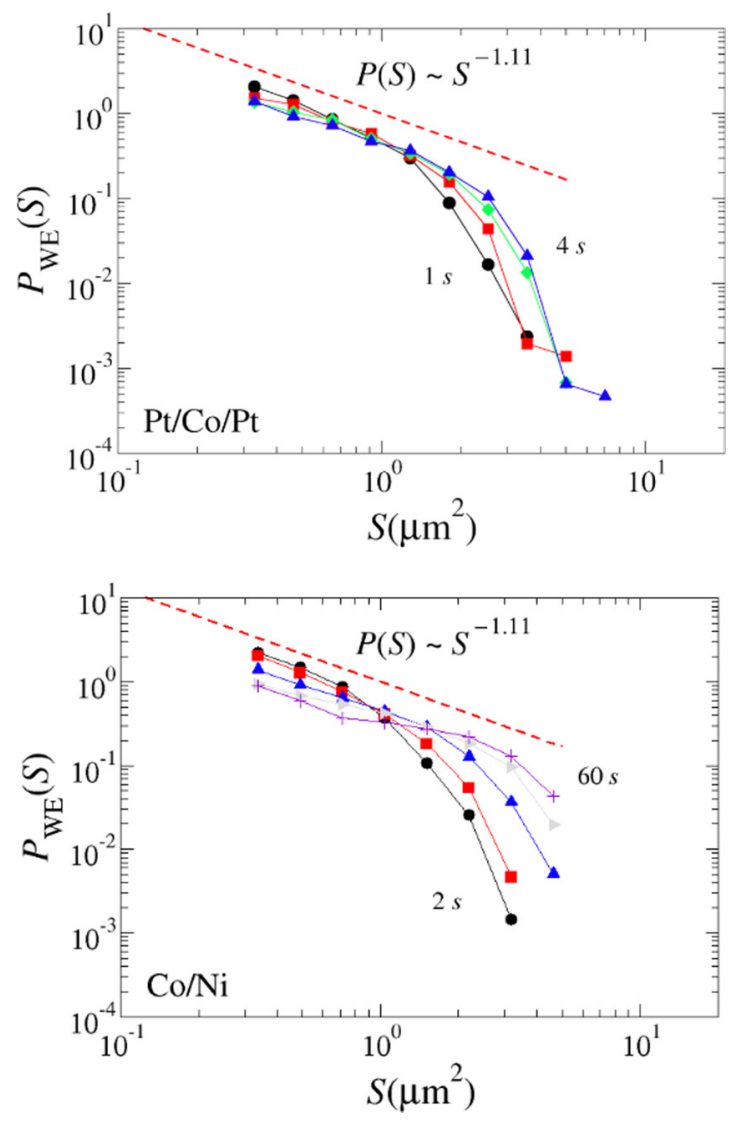

FIG. 9. WE area histograms obtained from a different region (upper panel) of the same $\operatorname{Pt}(4.5 \mathrm{~nm}) / \mathrm{Co}(0.7 \mathrm{~nm}) / \mathrm{Pt}(3.5 \mathrm{~nm})$ sample used to report most our results in the main text, and obtained for a different $\mathrm{Pt}(6 \mathrm{~nm}) /[\mathrm{Co}(0.2 \mathrm{~nm}) / \mathrm{Ni}(0.6 \mathrm{~nm})]_{3} / \mathrm{Al}(5 \mathrm{~nm})$ sample (lower panel). is not sensible to the tails. This justifies our event-detection protocol.

To evidence the robustness of our results, in Fig. 9 we show results for the event area distribution measurements done in a different region of the same $\mathrm{Pt}(4.5 \mathrm{~nm}) / \mathrm{Co}(0.7 \mathrm{~nm}) / \mathrm{Pt}(3.5 \mathrm{~nm})$ sample (upper panel), and for a $\operatorname{Pt}(6 \mathrm{~nm}) /[\mathrm{Co}(0.2 \mathrm{~nm}) / \mathrm{Ni}(0.6 \mathrm{~nm})]_{3} / \operatorname{Al}(5 \mathrm{~nm})$ sample (lower panel). As can be appreciated, not only the effective power-law decay exponent is similar, but also the time dependence is qualitatively similar to the one of Fig. 3 reported in Sec. III for the sample and region we have chosen for most of our analysis.

\section{APPENDIX B: DOMAIN-WALL ROUGHNESS}

To have a more direct estimate of the roughness exponent of our DWs and check consistency with our interpretation of the WE statistics, we have computed the single-value displacement field $u_{L}(x)$ of segments of given sizes $L$ partitioning a larger DW configuration. Here we discuss our practical method. The displacements $u_{L}(x)$ for each segment are measured with respect to the straight line fitting each segment. This straight line is also used as the $x$ axis to parametrize the displacement field. Such an approach is justified by taking into account that the theoretical description of a directed driven interface assumes that the interface is flat, on average, in the direction perpendicular to the motion. The field on magnetic DWs, on the other hand, acts as a pressure, always normal to the DW. Having $u_{L}(x)$ for segments of different length $L$, we can now compute their global squared width $W^{2}(L) \equiv$ $\overline{u_{L}^{2}(x)}-{\overline{u_{L}(x)}}^{2}$. If the interface is self-affine, we expect $W^{2} \sim L^{2 \zeta}$ with a well-defined roughness exponent $\zeta$. We have tested this methodology numerically on large discretized interfaces of size $L_{0}$, with displacement field $U(x)$, where $x=$ $0,1, \ldots, L_{0}-1$, with different precise values of $\zeta$. We do so by superimposing Fourier modes $U(x)=\sum_{q} U_{q} e^{i q x}$ with $q=2 \pi n / L_{0} \quad\left(n=0, \ldots, L_{0}-1\right), U_{q}$ complex Hermitian

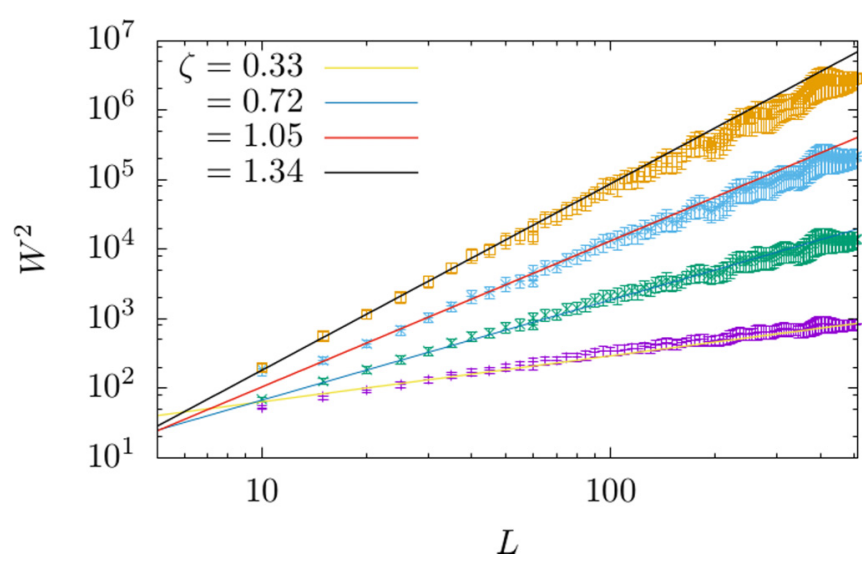

FIG. 10. Numerical test for the practical implementation used to compute $W^{2}$ for a DW. We compute $W^{2}$ for numerically generated self-affine Gaussian signals of size $L_{0}=1024$ for several precise values of $\zeta$. We average over ten samples for each $\zeta$. The solid lines show agreement with the expected $W^{2} \sim L^{2 \zeta}$. The method allows us to measure super-rough cases $\zeta>1$. 
Gaussian amplitudes of zero mean, $\left\langle U_{q}\right\rangle=0$, and variance $\left\langle\left|U_{q}\right|^{2}\right\rangle \sim 1 / q^{1+2 \zeta}$ (also known as self-affine Gaussian signals [36]). This construction assures that the signal $U(x)$ is periodic, $U(x)=U\left(x+L_{0}\right)$, and self-affine with identical spectral and global exponent $\zeta$. Finally, we compute $W^{2}(L)$ for these interfaces, by the partition procedure previously described. In Fig. 10, we compare $W^{2}(L)$ vs $L$ with the corresponding scalings for each $\zeta$, averaged over ten uncorrelated numerically sampled configurations $U(x)$. A good agreement is always obtained if the fit does not include values of $L$ larger than a fixed fraction of the order of the total size $L_{0}$, so to have a large number of segments and to reduce boundary effects. It is worth noting that our method also allows us to accurately measure values $\zeta>1$, corresponding to super-rough interfaces. This is an advantage over the displacement correlator function $B(x) \equiv \int_{0}^{L_{0}-x} d x_{0} \overline{\left[u\left(x+x_{0}\right)-u\left(x_{0}\right)\right]^{2}} /\left(L_{0}-\right.$ $x$ ), which gives the correct global $\zeta, B(x) \sim x^{2 \zeta}$, only if $\zeta<1$, otherwise it saturates to $\zeta=1$.
[1] S. Lemerle, J. Ferré, C. Chappert, V. Mathet, T. Giamarchi, and P. Le Doussal, Phys. Rev. Lett. 80, 849 (1998).

[2] P. J. Metaxas, J. P. Jamet, A. Mougin, M. Cormier, J. Ferré, V. Baltz, B. Rodmacq, B. Dieny, and R. L. Stamps, Phys. Rev. Lett. 99, 217208 (2007).

[3] K.-J. Kim, J.-C. Lee, S.-M. Ahn, K.-S. Lee, C.-W. Lee, Y. J. Cho, S. Seo, K.-H. Shin, S.-B. Choe, and H.-W. Lee, Nature 458, 740 (2009).

[4] J. Gorchon, S. Bustingorry, J. Ferré, V. Jeudy, A. B. Kolton, and T. Giamarchi, Phys. Rev. Lett. 113, 027205 (2014).

[5] V. Jeudy, A. Mougin, S. Bustingorry, W. Savero Torres, J. Gorchon, A. B. Kolton, A. Lemaître, and J.-P. Jamet, Phys. Rev. Lett. 117, 057201 (2016).

[6] R. Diaz Pardo, W. Savero Torres, A. B. Kolton, S. Bustingorry, and V. Jeudy, Phys. Rev. B 95, 184434 (2017).

[7] P. Le Doussal, K. J. Wiese, S. Moulinet, and E. Rolley, Europhys. Lett. 87, 56001 (2009).

[8] S. Atis, A. K. Dubey, D. Salin, L. Talon, P. Le Doussal, and K. J. Wiese, Phys. Rev. Lett. 114, 234502 (2015).

[9] O. Chepizhko, C. Giampietro, E. Mastrapasqua, M. Nourazar, M. Ascagni, M. Sugni, U. Fascio, L. Leggio, C. Malinverno, G. Scita, S. Santucci, M. J. Alava, S. Zapperi, and C. A. M. La Porta, Proc. Nat. Acad. Sci. 113, 11408 (2016).

[10] S. S. P. Parkin, M. Hayashi, and L. Thomas, Science 320, 190 (2008).

[11] T. Nattermann, Phys. Rev. Lett. 64, 2454 (1990).

[12] L. B. Ioffe and V. M. Vinokur, J. Phys. C 20, 6149 (1987).

[13] G. Durin, F. Bohn, M. A. Corrêa, R. L. Sommer, P. Le Doussal, and K. J. Wiese, Phys. Rev. Lett. 117, 087201 (2016).

[14] L. Laurson, X. Illa, S. Santucci, K. Tore Tallakstad, K. J. Måløy, and M. J. Alava, Nat. Commun. 4, 2927 (2013).

[15] V. Repain, M. Bauer, J.-P. Jamet, J. Ferré, C. Chappert, and H. Bernas, Europhys. Lett. 68, 460 (2004).

[16] E. E. Ferrero, L. Foini, T. Giamarchi, A. B. Kolton, and A. Rosso, Phys. Rev. Lett. 118, 147208 (2017).

[17] P. Chauve, T. Giamarchi, and P. Le Doussal, Phys. Rev. B 62, 6241 (2000).

[18] J.-P. Bouchaud and A. Georges, Phys. Rep. 195, 127 (1990).
[19] V. H. Purrello, J. L. Iguain, A. B. Kolton, and E. A. Jagla, Phys. Rev. E 96, 022112 (2017).

[20] E. E. Ferrero, S. Bustingorry, and A. B. Kolton, Phys. Rev. E 87, 032122 (2013).

[21] A. Rosso and W. Krauth, Phys. Rev. Lett. 87, 187002 (2001).

[22] L.-H. Tang, M. Kardar, and D. Dhar, Phys. Rev. Lett. 74, 920 (1995).

[23] A.-L. Barabási and H. E. Stanley, Fractal Concepts in Surface Growth (Cambridge University Press, Cambridge, 1995).

[24] A. B. Kolton, A. Rosso, T. Giamarchi, and W. Krauth, Phys. Rev. Lett. 97, 057001 (2006).

[25] A. B. Kolton, A. Rosso, T. Giamarchi, and W. Krauth, Phys. Rev. B 79, 184207 (2009).

[26] S. Zapperi, P. Cizeau, G. Durin, and H. E. Stanley, Phys. Rev. B 58, 6353 (1998).

[27] M. Kardar, Phys. Rep. 301, 85 (1998).

[28] V. M. Vinokur, M. C. Marchetti, and L.-W. Chen, Phys. Rev. Lett. 77, 1845 (1996).

[29] C. Monthus and T. Garel, Phys. Rev. E 78, 041133 (2008).

[30] S. Emori and G. S. D. Beach, J. Phys. Condens. Matter 24, 024214 (2012).

[31] K.-W. Moon, D.-H. Kim, S.-C. Yoo, C.-G. Cho, S. Hwang, B. Kahng, B.-C. Min, K.-H. Shin, and S.-B. Choe, Phys. Rev. Lett. 110, 107203 (2013).

[32] A. Hrabec, N. A. Porter, A. Wells, M. J. Benitez, G. Burnell, S. McVitie, D. McGrouther, T. A. Moore, and C. H. Marrows, Phys. Rev. B 90, 020402(R) (2014).

[33] A. W. J. Wells, P. M. Shepley, C. H. Marrows, and T. A. Moore, Phys. Rev. B 95, 054428 (2017).

[34] J.-C. Rojas-Sánchez, P. Laczkowski, J. Sampaio, S. Collin, K. Bouzehouane, N. Reyren, H. Jaffrès, A. Mougin, and J.-M. Georges, App. Phys. Lett. 108, 082406 (2016).

[35] N. B. Caballero, I. Fernández Aguirre, L. J. Albornoz, A. B. Kolton, J. C. Rojas-Sánchez, S. Collin, J. M. George, R. Diaz Pardo, V. Jeudy, S. Bustingorry, and J. Curiale, Phys. Rev. B 96, 224422 (2017).

[36] A. Rosso, R. Santachiara, and W. Krauth, J. Stat. Mech.: Theor. Exp. (2005) L08001. 